\title{
Xavier Leroux, André Janson et Bernard Malczyk, 2014 (reed.), Géographie à vivre - CM1
}

5 modules pour acquérir des concepts et des savoir-faire, ACCES Editions, Schiltigheim, 144 pages, 3 cartes grand format en annexe, 1 DVD

Nicolas Lebrun

\section{(2) OpenEdition}

Electronic version

URL: http://journals.openedition.org/rge/5122

DOI: $10.4000 /$ rge.5122

ISSN: 2108-6478

\section{Publisher}

Association des géographes de l'Est

Printed version

Date of publication: 30 December 2013

ISSN: 0035-3213

\section{Electronic reference}

Nicolas Lebrun, « Xavier Leroux, André Janson et Bernard Malczyk, 2014 (reed.), Géographie à vivre CM1 », Revue Géographique de l'Est [Online], vol. 53 / 3-4 | 2013, Online since 17 July 2014, connection on 23 September 2020. URL : http://journals.openedition.org/rge/5122 ; DOI : https://doi.org/ $10.4000 /$ rge. 5122

This text was automatically generated on 23 September 2020.

Tous droits réservés 


\title{
Xavier Leroux, André Janson et Bernard Malczyk, 2014 (reed.), Géographie à vivre - CM1
}

5 modules pour acquérir des concepts et des savoir-faire, ACCES Editions, Schiltigheim, 144 pages, 3 cartes grand format en annexe, 1 DVD

\author{
Nicolas Lebrun
}

\section{REFERENCES}

Xavier Leroux, André Janson et Bernard Malczyk, 2014 (reed.), Géographie à vivre - CM1, 5 modules pour acquérir des concepts et des savoir-faire, ACCES Editions, Schiltigheim, 144 pages, 3 cartes grand format en annexe, 1 DVD

1 L'enseignement de la géographie à l'école primaire ne fait pas véritablement l'objet d'attentions particulières de la part des éditeurs. Or, la faible proportion de professeurs des écoles ayant reçu une formation disciplinaire en géographie, combinée à des programmes riches et difficiles à mettre en œuvre, contribuent à générer une réelle attente. En effet, la discipline géographique plus que d'autres parfois, est assez difficile d'approche, du fait de son vocabulaire spécifique bien entendu, mais aussi des postures particulières à acquérir lorsqu'on étudie l'espace géographique.

2 Dès lors, l'initiative lancée en 2011 par ACCES Editions de confier à des géographes la réalisation d'ouvrages destinés aux professeurs des écoles désireux d'améliorer l'enseignement de la géographie dans les classes, ne pouvait être que saluée. Ces ouvrages font tous l'objet d'une nouvelle édition en 2014.

3 Les auteurs, Xavier Leroux, professeur des écoles et docteur en géographie, André Janson et Bernard Malczyk, formateurs à l'École Supérieure du Professorat et de l'Éducation (ESPE) Lille Nord de France, ont, grâce à leur connaissance du milieu scolaire, conçu une série d'ouvrages conformes aux attentes des enseignants du primaire. Il ne s'agit pas d'ouvrages destinés aux élèves mais bel et bien d'ouvrages 
conçus pour des enseignants, géographes ou non de formation. Ils se caractérisent par leur richesse documentaire et le développement d'activités destinées au niveau dédié, le tout dans une approche simple et claire.

4 Le plan de chaque ouvrage est construit en fonction des programmes scolaires en vigueur, en rappelant la répartition des points traités entre les différents tomes. Il s'agit toujours de mettre l'enseignant en position de comprendre les enjeux géographiques liés à l'enseignement d'un point du programme, de lui suggérer des constructions d'ensemble documentaires, de lui fournir des documents vierges à compléter en classe (présents dans l'ouvrage et sur CD pour impression et photocopies). $\mathrm{Au}$ regard de la pertinence des mises en situation professionnelle (élaboration de croquis paysager, enquête à réaliser, légende à renseigner, etc.), les activités proposées permettent à l'enseignant généraliste de mieux comprendre les attentes de la discipline. Cela lui permet non seulement de faire face dans l'urgence à certaines situations d'enseignements - c'est là un usage récurrent des manuels destinés aux enseignants - mais aussi de lui donner les moyens d'être capable de devenir, de luimême et par la suite, producteur d'activités attrayantes conformes aux enjeux de la Géographie.

5 Notons que les ouvrages de la collection «Géographie à vivre » existent pour les 3 niveaux du cycle 3 (CE2, CM1, CM2). Nous nous sommes intéressés plus spécifiquement à l'ouvrage destiné au niveau CM1. Mais si la géographie n'est pas présente en tant que discipline scolaire en cycle 1 et 2 , les repères spatiaux permettent déjà d'aborder la réflexion géographique. De fait on retrouvera de mêmes démarches et attentes dans la collection «Temps et espace à vivre» destinés aux CP, CE1 niveaux auxquels l'apprentissage du repérage dans l'espace suppose des compétences spécifiques, alors que les notions d'échelle et de perspective ne sont pas encore réellement maitrisées.

\section{AUTHORS}

\section{NICOLAS LEBRUN}

Maître de conférences en géographie - Laboratoire Discontinuités, EA 2468 - Université Lille Nord de France, Artois - nicolas.lebrun@univ-artois.fr 